\title{
A Study into Digital Dermatitis Transmission and Bacterial Associated Pathological Changes Involved in the Disease
}

\author{
N. Capion ${ }^{1 *}$, M. Boye ${ }^{2}$, C. Ekstrøm ${ }^{3}$, K. Dupont ${ }^{2}$, T. K. Jensen ${ }^{2}$ \\ ${ }^{1}$ Department of Large Animal Sciences, Faculty of Health and Medical Sciences, University of Copenhagen, Copenhagen, Denmark \\ ${ }^{2}$ National Veterinary Institute, Technical University of Denmark, Copenhagen, Denmark \\ ${ }^{3}$ Department of Biostatistics, University of Southern Denmark, Odense, Denmark \\ Email: "nyc@sund.ku.dk
}

Received February 23, 2013; revised March 23, 2013; accepted April 23, 2013

Copyright (C) 2013 N. Capion et al. This is an open access article distributed under the Creative Commons Attribution License, which permits unrestricted use, distribution, and reproduction in any medium, provided the original work is properly cited.

\begin{abstract}
Digital dermatitis is a highly prevalent painful lesion affecting the feet in dairy cattle. Even though the pathogenesis has been subject of investigation since 1974, there is still a lack of knowledge about the spread of the disease among cows within a herd as well as between herds. The purpose of this study was to monitor transmission of digital dermatitis under experimental conditions between naïve heifers and affected animals, to monitor the changes in clinical appearance, microbial colonisation of the skin as lesions progressed and to apply a q-PCR for the detection of Treponema spp. in faecal samples. Eight heifers with clinical normal digital skin were housed with 5 heifers with severe digital dermatitis lesion for 8 weeks on a solid concrete floor with an accumulating layer of slurry. Digital skin was examined daily and lesions were clinically scored. Skin biopsies were taken from the healthy heifers at introduction and weekly from all lesions for histopathological evaluation and fluorescence in situ hybridization. None of the healthy heifers developed digital dermatitis and in 4 out of 5 infected heifers the lesions healed during the study. All samples from healthy skin were negative for Treponema spp. and one sample were positive for Dichelobacter nodosus. Colonization of healthy skin could not be identified in this study. There was no significant relation between clinical scoring of the lesions and histopathological score and the presence of Treponema spp. There were however a significant relation between the prevalence of Treponema spp. in the skin and severity of changes in epidermis and dermis. By qPCR all the healthy heifers were found to excrete Treponema spp. in their faeces.
\end{abstract}

Keywords: Digital Dermatitis; Treponema spp.; Histopathology; Infection Dynamics

\section{Introduction}

Digital dermatitis (DD) is the most common cause of lameness in Danish dairy herds. A study performed in 2003 showed that $89 \%$ of Danish Holstein dairies have cows infected with DD and the mean herd level prevalence was $21 \%$ [1].

The pathogenesis of DD has been intensely investigated during the last decades. DD appears to be polymicrobial, since a wide variety of bacteria have been isolated from lesions [2,3]. Recently laboratory techniques, has made the identification of bacteria such as spirochetes possible within the epidermis and dermis using cultureindependent methods. More than 16 different Treponema phylotypes have been identified in DD lesions. The most commonly found bacteria in association with DD are treponemes belonging to three different clusters $T$. phage-

${ }^{*}$ Corresponding author. denis-like [4-7], T. refringens-like [8,9] and T. denticola-like [10]. Even though the microbiological background for DD has been intensely investigated; there is still uncertainty about the spread of the disease. The ability of the causative agents to survive in the environment is unknown and therefore the knowledge of contamination within a herd is yet to be elucidated. DD has been called contagious and infectious [11], however, experimental transmission is difficult [12]. Only two times has an experimental spread of DD been reported as successful. Both studies had to macerate the skin and have anaerobic conditions unlike conditions seen with natural infection $[13,14]$.

It is obvious from clinical studies and reports of outbreaks that DD do spread among cows in a herd; however the routes of disease spreading have not been elucidated. The development of the lesion and colonization of the pathogens in the skin prior to clinical DD have not 
been investigated, which means that the initial causative agents have not been identified. Studies have examined healthy skin in the perimeter of lesions and have not been able to show the presence of Treponema spp. [8]. Several studies have described finding Treponema spp. in the GI tract of bovine [15-18] and these treponemes are probably shed in feces, however the treponemes found associated with digital dermatitis have not been isolated from GI samples or feces [15]. The reservoir for DD Treponemes remains uncertain.

Naïve young stock/young heifers have previously been described to be especially susceptible to DD [19-21] and moisture and detrimental effect of urine and faeces are known risk factors for DD $[21,22]$.

The purpose of this study was 1) to monitor transmission of digital dermatitis under experimental conditions between naïve heifers and affected animals; 2) to evaluate the relation between clinical macroscopic appearance (lesion stage) of DD and histopathological changes and microbial colonization of the skin and 3) to apply a qPCR for the detection of Treponema spp. in faecal samples.

\section{Materials and Methods}

\subsection{Animals}

Two maiden Holstein heifers 13 months of age from a DD-free tie stall herd (herd 1) and six maiden Holstein heifers 15 months of age from a DD-free free stall herd (herd 2) were housed together in a stall with solid concrete floor that measured $14 \mathrm{~m}$ by $8 \mathrm{~m}$. Twelve hours a day the heifers has access to an additional area covered with deep straw bedding measuring $6 \mathrm{~m}$ by $8 \mathrm{~m}$. During a period of four weeks the eight heifers build up a layer of approximately $40 \mathrm{~cm}$ of slurry on the concrete floor. After the four week introduction-period one first lactation Holstein cow (herd 3) with clinical lameness score $4 / 5$ [23] and DD on both hind legs, from an endemic infected free stall herd, were introduced to the eight heifers. Following another four week period four maiden Holstein heifers (herd 4) from an endemic infected free stall herd were introduced to the group. All heifers had severe DD in the hind legs and a lameness score of $3 / 5$ or $4 / 5$ [23].

\subsection{Examination}

Before introduction to the experimental housing facility the heifers were examined in a trimming chute. Photographs were taken of all four limbs, the claws were trimmed and all lesions were recorded. Punch biopsies (6 $\mathrm{mm}$, Kruuse, Denmark) were taken in the digital skin proximal of the heel horn on all hind legs following local anaesthesia of the skin, with $10 \mathrm{ml}$ of Lidocain (20 $\mathrm{mg} / \mathrm{ml})$. No treatment or bandage was applied following biopsy sampling.

After introduction to the group all animals were examined on a daily basis. The examination included evaluation of general appearance, temperature, heart rate, respiratory rate, rumen contractions, faeces consistency, hydration, and they received a lameness score and were palpated for soreness of the digital skin. Once a week the heifers were examined in the trimming chute. The examination in the trimming chute included inspection and palpation of the digital skin. Faeces were removed with a gloved hand simultaneously with the palpation, leaving the skin visible but not clean, skin/lesions were scored for lesion stage and biopsies were sampled from all lesions.

\subsection{Classification of DD Lesion Stage}

DD was recorded using a standardised scoring system comprising five stages (M0-M4) [24]. If different stages of DD were present, the lesion was scored according to the most predominant stage of DD. M0: Normal skin, M1: Early stage of DD with a circumscribed granulomatous area, 0 to $4 \mathrm{~cm}$ in diameter, which lies at the epithelial surface or up to $2 \mathrm{~mm}$ underneath it. M1 is generally not painful on palpation. M2: Classical ulcerative stage of $\mathrm{DD}$, with granulomatous tissue. M2 is often painful on palpation. M3: Classical ulceration of DD in the process of healing covered by a scab. M3 is generally not painful on palpation. M4: The chronic stage of DD are dyskeratotic or hyperkeratotic and can present themselves with a proliferative aspect. M4 is generally not painful on palpation.

\subsection{Biopsies}

Biopsies were fixed in 10\% neutral buffed formalin, dehydrated and embedded in paraffin blocks. Serial tissue sections $(4 \mu \mathrm{m})$ were cut and mounted on Super Frost + slides (Menzel-Gläser, Germany) for haematoxylin and eosin (H\&E) stain and fluorescence in situ hybridization (FISH).

\subsection{Histopathological Evaluation}

All samples were evaluated histopathologically in H\&E stained sections. Epidermal changes were scored 0 to 4 $(0=$ normal epidermis, $1=$ hyperkeratosis and epidermal hyperplasia, $2=$ focal, $3=$ moderate, and $4=$ severe acanthotic epidermis with or without parakeratotic papillomatous proliferation and ballooning degeneration of keratinocytes, exocytosis and/or erosion of dermal papilla).

Additionally, the biopsies were graded according to the inflammatory response in dermis as mild or absent (score 1), moderate (score 2), or (score 3) severe. 


\subsection{Fish}

The biopsies were hybridized using oligonucleotide probes specific for Domain bacterium, Dichelobacter nodosus; Treponema phagedenis as well as Treponema spp.; (a Treponema group probe) as previously reported [8].

The hybridized sections were all read and scored by one of the senior authors. The total bacterial (probe for Domain bacterium) colonization of the epidermis was scored from 0 to $3(0=$ no invasive bacteria, $1=$ low number of invasive bacteria, $2=$ moderate number of bacteria, and 3 = high number of invasive bacteria). Similarly, the prevalence Treponema spp. and T. phagedenis-like organisms were scored from 0 to 3 , whereas the prevalence of $D$. nodosus was scored $0=$ no hybridization, or 1 = positive hybridization.

\subsection{Collection of Fecal Samples and DNA Extraction}

Forty eight samples of faeces were collected from the 8 heifers from herd 1 and 2 during 6 consecutive days (one sample per animal per day). The samples were frozen immediately and stored at $-20^{\circ} \mathrm{C}$.

DNA was extracted from the samples using the QIAamp DNA Stool Mini Kit (QIAGEN, Hilden, Germany): 180 $220 \mathrm{mg}$ of frozen faeces was transferred to ASL lysis buffer and DNA was extracted following manufacturer's protocol. The purified DNA was quantified (the yield per sample was approximately $6 \mu \mathrm{g}$ with no variation between the samples) and diluted in nuclease-free water (Applied Biosystems, Foster City, CA, USA) to the concentration of $6.5 \mathrm{ng} \times \mu \mathrm{L}^{-1}$.

DNA for standard serial dilutions was obtained from a Treponema phagedenis isolate (isolate VI) provided by the Dept. of Biomedical Sciences and Veterinary Public Health, Swedish University of Agricultural Sciences, Uppsala, Sweden.

DNA was extracted using Easy-DNA Kit, protocol \#3 (Invitrogen, Taastrup, Denmark). The quality of the purified DNA was verified both photometrically using a (NanoDrop 2000) spectrophotometer (Thermo Scientific, Wilmington. DE, USA) and by performing a PCR with general and Treponema spp. specific 16S RNA gene primers.

\section{8. qPCR}

The serial dilutions with well-defined number of DNA copies were created by cloning Treponema phagedenis 16S RNA gene fragment into pCR4Blunt-TOPO vector (Zero Blunt $^{\circledR}$ TOPO $^{\circledR}$ PCR Cloning Kit for Sequencing, Invitrogen)

PCR was performed in $4 \times 50 \mu \mathrm{L}$ reaction mixture containing: $0.5 \mu \mathrm{M}$ of each primer, dNTP (each nucleo- tide $400 \mu \mathrm{M}), \mathrm{MgSO}_{4}(2.0 \mathrm{mM})$, pfu DNA polymerase (0.05 $\mathrm{U} \times \mu \mathrm{L}^{-1}$; Promega Corporation, Madison, WI, USA), template DNA from Treponema phagedenis isolate (40 ng per $50 \mu \mathrm{L}$ of mix).

The cloning was performed according to the Invitrogen protocol for Zero Blunt ${ }^{\circledR}$ TOPO $^{\circledR}$ PCR Cloning Kit. Five selected transformants were cultured overnight, harvested and the plasmids were purified using Plasmid Mini Kit (Qiagen, Cat. No.12123). The purified plasmids were quantified (Nanodrop) and digested with Pst I (Promega, cat. no. R4114) following Promega's protocol. The plasmid digest was then purified with MinElute PCR Purification. The insert sequence was controlled by sequencing purified plasmid using the Treponema primers. Sequencing was performed on a 3130 Genetic Analyzer (Applied Biosystems, USA) using BigDye Terminator v3.1 Cycle Sequencing Kit (Applied Biosystems, cat. no. 4337455) according to the manufacturer's protocol. The samples were run in triplicates.

qPCR was performed using a Rotor-Gene 6000 (Corbett Research, Sydney, Australia).

For qPCR on DNA extracted from faeces qPCR was run as single reactions for standard dilution and as triplicates for the samples. The conditions of PCR were the same as described above.

\subsection{Statistical Analyses}

To determine whether clinical classification of lesion stage, changes in epidermis and, inflammatory response in dermis were influenced by the prevalence of Treponema spp., prevalence of $T$. phagedenis-like, and prevalence of $D$. nodosus a multinomial (ordered) regression model was employed. The model was fitted using generalized estimating equations (GEE) with a working independence covariance structure based on the specific lesion within cow in order to accommodate any effect of repeated observations on a single lesion. Misspecification of the correct correlation structure was not critical with respect to the fixed effect estimates and would result in a loss of efficiency. Clusters defined by leg within cow were considered to see if this influenced the standard error of the estimated parameters.

Due to the size of the dataset and the low number of animals with DD a forward selection scheme was used to identify the associated predictors and the biological effect of the associated predictors was subsequently quantified.

\subsection{Generalized Estimation Equation}

For the generalized estimating equation (GEE) procedure, a multinomial error distribution and a cumulative logistic link function was employed. Initially, a working independence covariance structure with clusters based on 
individual legs was used. However, clusters based on individual lesions or on the broader grouping defined by cows were also considered. Explanatory variables using a forward selection approach was included and subsequently the full model was reduced using model reduction.

\section{Results}

None of the 8 naïve heifers developed digital skin lesions during the 12 week observation period. The two heifers from tie stall (herd 1) developed hyperkeratosis of the digital skin above the heel bulbs. There were one case of lameness due to distortion and swelling of the fetlock joint on a foreleg. Otherwise the naïve heifers remained healthy. However, one biopsy was positive for Dichelobacter nodosus in the final biopsy sample collected from one of the healthy heifers at the end of the 12th week. The clinical evaluation of the skin showed hyperkeratosis and this was confirmed in the histology sample upon analysis.

Table 1 shows the status of DD lesions during the 12 weeks of study. The first lactation cow (herd 3) introduced after four weeks had DD on both hind legs. On left hind leg there was a lesion on an interdigital hyperplasia and a lesion between the heel bulbs. On the right hind leg there was a lesion between and above the heel bulbs. Both lesions between and above the heel bulbs healed completely, during the eight weeks in the experimental setting, where the legs were constantly covered in manure. However, the DD on the interdigital skin remained throughout the study. Three of the four heifers from herd 4 , had lesions that healed completely during the study period.

Table 1. Total number of digital dermatitis lesions on the individual heifers from introduction to the study population to the end of the study period.

\begin{tabular}{|c|c|c|c|c|c|c|c|c|c|c|c|c|c|}
\hline \multirow{3}{*}{ Herd } & \multirow{3}{*}{ Cow } & \multicolumn{12}{|c|}{ Number of DD lesions } \\
\hline & & \multicolumn{12}{|c|}{ Week } \\
\hline & & 1 & 2 & 3 & 4 & 5 & 6 & 7 & 8 & 9 & 10 & 11 & 12 \\
\hline 1 & 1 & 0 & 0 & 0 & 0 & 0 & 0 & 0 & 0 & 0 & 0 & 0 & $0^{*}$ \\
\hline 1 & 2 & 0 & 0 & 0 & 0 & 0 & 0 & 0 & 0 & 0 & 0 & 0 & 0 \\
\hline 2 & 3 & 0 & 0 & 0 & 0 & 0 & 0 & 0 & 0 & 0 & 0 & 0 & 0 \\
\hline 2 & 4 & 0 & 0 & 0 & 0 & 0 & 0 & 0 & 0 & 0 & 0 & 0 & 0 \\
\hline 2 & 5 & 0 & 0 & 0 & 0 & 0 & 0 & 0 & 0 & 0 & 0 & 0 & 0 \\
\hline 2 & 6 & 0 & 0 & 0 & 0 & 0 & 0 & 0 & 0 & 0 & 0 & 0 & 0 \\
\hline 2 & 7 & 0 & 0 & 0 & 0 & 0 & 0 & 0 & 0 & 0 & 0 & 0 & 0 \\
\hline 2 & 8 & 0 & 0 & 0 & 0 & 0 & 0 & 0 & 0 & 0 & 0 & 0 & 0 \\
\hline 3 & 9 & & & & & 3 & 3 & 3 & 2 & 1 & 1 & 1 & 1 \\
\hline 4 & 10 & & & & & & & & & 3 & 3 & 3 & 2 \\
\hline 4 & 11 & & & & & & & & & 3 & 3 & 3 & 2 \\
\hline 4 & 12 & & & & & & & & & 1 & 1 & 1 & 1 \\
\hline 4 & 13 & & & & & & & & & 2 & 2 & 2 & 1 \\
\hline
\end{tabular}

*This sample had a positive hybridisation of $D$. nodosus.

\subsection{Treponema spp.}

Because of few observations for some categories, lesion stages M1 and M2, lesion stages M3 and M4, and change in epidermis score 0 and 1 were combined when analysing the prevalence of Treponema spp. The score for inflammation in dermis and for changes in epidermis were included in the full model as well as examination date. Classification of lesion stage was not significant $(\mathrm{p}=$ $0.18)$.

The final model included either changes in epidermis score $(\mathrm{p}=0.02)$ or inflammation in dermis score $(\mathrm{p}=$ 0.03 ) but not both. When individual legs were used as cluster the result were the same ( $p=0.04$ for changes in epidermis and $\mathrm{p}=0.02$ for inflammation in dermis).

Table 2 shows that a moderate inflammatory response in dermis (score 2) had an odds ratio resulting in a lower prevalence number of Treponema spp. of 0.56 relative to an extensive to diffuse inflammatory response in dermis (score 3) (i.e. odds ratio of 1.78 for a higher prevalence of Treponema spp.). The odds ratio of absent or mild inflammatory response in dermis (score 1) (relative to 3) was 7.63 for a lower prevalence of Treponema spp.

The results from the final model for the prevalence of Treponema spp. including changes in epidermis (Table 3) show that changes in epidermis score 2 had an odds ratio resulting in a lower prevalence of Treponema spp. of 62.85 relative to more severe changes in epidermis (score 3 ). The odds ratio of category 1 (relative to 3 ) was 355.28 for a lower prevalence of Treponema spp. These extremely large odds ratios were due to the small size of the dataset relative to the number of categories.

Table 2. The estimates for the final model for the prevalence of Treponema spp. including inflammation in dermis score 1,2 and 3.

\begin{tabular}{ccccccc}
\hline Parameter & Estimate & $\begin{array}{c}\text { Standard } \\
\text { Error }\end{array}$ & \multicolumn{2}{c}{ 95\% Confidence } & Limits & $\mathrm{Zr}>|\mathrm{Z}|$ \\
\hline Intercept 1 & -2.11 & 1.028 & -4.11 & -0.12 & -2.07 & 0.04 \\
Intercept 2 & -1.83 & 0.97 & -3.72 & 0.06 & -1.90 & 0.06 \\
Intercept 3 & -0.919 & 1.15 & -3.17 & 1.35 & -0.79 & 0.43 \\
Score 1 & 2.03 & 0.75 & 0.57 & 3.49 & 2.72 & 0.01 \\
Score 2 & -0.58 & 1.12 & -2.78 & 1.63 & -0.51 & 0.61 \\
Score 3 & 0.00 & 0.00 & 0.00 & 0.00 & & \\
\hline
\end{tabular}

Table 3. The estimates for the final model for the prevalence of Treponema spp. including changes in epidermis score 1,2 and 3.

\begin{tabular}{ccccccc}
\hline Parameter & \multirow{2}{*}{ Estimate } & $\begin{array}{c}\text { Standard } \\
\text { Error }\end{array}$ & \multicolumn{2}{c}{$\begin{array}{c}\text { 95\% Confidence } \\
\text { Limits }\end{array}$} & $\mathrm{Z}$ & $\mathrm{Pr}>|\mathrm{Z}|$ \\
\hline Intercept 1 & -4.54 & 0.56 & -5.63 & -3.45 & -8.17 & $<0.01$ \\
Intercept 2 & -4.06 & 0.81 & -5.65 & -2.48 & -5.03 & $<0.01$ \\
Intercept 3 & -2.16 & 0.71 & -3.55 & -0.77 & -3.04 & $<0.01$ \\
Score 1 & 5.87 & 1.12 & 3.68 & 8.07 & 5.24 & $<0.01$ \\
Score 2 & 4.14 & 1.23 & 1.73 & 6.55 & 3.36 & 0.01 \\
Score 3 & 0.00 & 0.00 & 0.00 & 0.00 & & \\
\hline
\end{tabular}




\subsection{Treponema phagedenis-Like Organisms}

Bnges in epidermis categories 0 and 1 were combined when analysing $T$. phagedenis.

The main effect of inflammation in dermis score and changes in epidermis score were both significant ( $p$-values of 0.02 and 0.03 ) while macroscopically classification of DD was not $(p=0.07)$. However, the size of the data did not allow for an inclusion of multiple explanatory variables, and even individual legs as clusters were considered there was no significance.

The estimates for the classification of lesion stage main effects are shown below (Table 4) although the overall effect was not significant.

\subsection{Dichelobacter nodosus}

There were only two possible categories for $D$. nodosus so a logistic regression model was used with a cluster that is defined either by leg or lesion.

In order to be able to estimate the parameters the changes in epidermis scores 0 and 1 , as well as lesion stages M3 and M4 were grouped. The single observation belonging to the healthy individual with M0 was disregarded. Inflammation in dermis score could not be used directly in a logistic regression setting as there was only observed variation for score 1 . Consequently, $D$. nodosus and inflammation in dermis score were compared using Fisher's exact test (which on the other hand disregarded the fact that there was repeated measurements on the individuals). The size of the data did not allow for inclusion of multiple explanatory variables, which left only the main effects of the remaining two explanatory variables of interest.

Neither changes in epidermis score $(p=0.89)$ nor classification of lesion stage were significant $(p=0.09)$. However, looking at changes in epidermis and examination data combined then examination date was borderline significant $(\mathrm{p}=0.04)$.

Only one of the analyses showed any systematic effect of time although measurements taken on the same cluster were potentially positively correlated. The statistical effect, however, was very small. It should be stressed that the number of observations was quite small for this study

Table 4. The main effects of DD lesion stage (M2, M3 and M4) on the prevalence of $T$. phagedenis.

\begin{tabular}{ccccccc}
\hline Parameter & Estimate & $\begin{array}{c}\text { Standard } \\
\text { Error }\end{array}$ & $\begin{array}{c}\text { 95\% Confidence } \\
\text { Limits }\end{array}$ & $\mathrm{Z}$ & $\operatorname{Pr}>|\mathrm{Z}|$ \\
\hline Intercept 1 & -2.62 & 0.55 & -3.70 & -1.54 & -4.77 & $<0.01$ \\
Intercept 2 & -2.36 & 0.48 & -3.29 & -1.43 & -4.96 & $<0.01$ \\
Intercept 3 & -0.83 & 0.69 & -2.18 & 0.53 & -1.19 & 0.23 \\
M2 & 0.17 & 1.31 & -2.41 & 2.74 & 0.13 & 0.90 \\
M3 & 2.95 & 0.41 & 2.14 & 3.76 & 7.13 & $<0.01$ \\
M4 & 0.00 & 0.00 & 0.00 & 0.00 & & \\
\hline
\end{tabular}

and given the number of categories for the response and explanatory variables so in most cases it had not been possible to make analyses that accommodate all the available predictors. It is also worth emphasizing that many of the results were just borderline significant (below the 5\% level), and that while they all point in the same direction (which suggests that the statistical significance might have been greater if the dataset had been larger), also keeping in mind that multiple test/analyses have been made.

\subsection{Treponema spp. qPCR on Faeces}

The standard dilutions $\mathrm{Ct}$ values were consistent with expected 10 folds concentration differences between consecutive standards and ranged from 7.18 for the $10^{7}$ standard dilution to 26.92 for the $10^{1}$ standard dilution. The $\mathrm{Ct}$ values for the samples lay between 14.73 and 18.05 .

The $\mathrm{t}_{\mathrm{m}}$ (melting temperature) for standard dilutions (Treponema phagedenis) was $86.5^{\circ} \mathrm{C}-86.8^{\circ} \mathrm{C}$. The $\mathrm{t}_{\mathrm{m}} \mathrm{s}$ for samples ranged from $84.5^{\circ} \mathrm{C}$ to $86.7^{\circ} \mathrm{C}$. The wider melting temperature range of the samples was most probably caused by the fact that this was a mixture of Treponema spp. therefore one would expect some variation in the amplified region of the 16S RNA gene.

The number of Treponema spp. copies in the samples was estimated by absolute quantification using standard serial dilutions. Treponema species were found in all samples with the number varying from 4000 to 38,000 copies. Although Treponema spp. was present in all samples there was no significant variation in the number of copies detected.

\section{Discussion}

Based on the result of this study, the experimental transfer of DD between heifers did not occur. The area the heifers were kept in was rather crowded, with approximately $8.6 \mathrm{~m}^{2}$ per animal. New animals were introduced to the group every 4 week, causing disturbance and probably introducing new pathogens. One might speculate that the environment the heifers were kept in probably did not particularly favour treponemes, since none of the naïve animals was infected. Another thought could be that the skin was exposed to urea instead of ammonium gas and thereby was in better condition to limit the DD lesions and the proliferation of treponemes within the epidermis. The possibility of the naïve heifers being unsusceptible to $\mathrm{DD}$ is also present. There has been some evidence that a rather large variation in susceptibility between Holstein heifers exists [25].

The PCR showed large amounts of treponemes in the faeces, hence in the manure covering the floor. Even though previous studies have failed to identify DD-tre- 
ponemes in the bovine GI-canal [15] and the present study failed to classify the exact Treponema spp. in the faecal samples it still poses the question whether the manure is in fact the reservoir for DD. The finding that DD did not spread in this group of animals, in spite of several risk factors supports the theory that DD indeed has a complicated multifactorial etiology.

However, it is quite interesting that despite the rather unhygienic conditions these heifers were exposed to for 12 weeks none of them developed signs of DD or even interdigital dermatitis or heel horn erosion. And epidermal colonisation of bacteria could only be found in one biopsy from hyperkeratotic skin.

With regards to the gradual healing of lesions in the group of infected cows, the frequent biopsy sampling could have induced an inflammatory response that acted synergistically with the cow and sped up the healing process. This completely adverse direction this part of the study has taken only shows that there are still many unknowns about this particular disease.

The most common way of diagnosing DD in practice is by clinically identifying the lesion and more advanced scoring the lesion according to a scoring system. The results show that the relationship between clinical appearance of the lesion, the lesion stage and the degree of histopathological changes or the degree of treponemal colonisation of the tissue is very poor. This is in contrast to the previous findings of this research group. In 2007 this group found that the larger and the more variable the colonisation the more severe were the lesion stage and the clinical evaluation based on data collected in a crosssectional study [8]. One may speculate that the DD lesions in the present study are atypical lesions due to the effect of the environment that actually improves or heals the lesions. In the present study we saw a discontinuation of a typical DD lesion instead of a development of new lesions. Furthermore data were based on repeated measurements on the same animals, providing more information from each lesion.

However, when analysing at the histopathological changes in epidermis and dermis there is a correlation between more treponemes and more severe changes in dermis and epidermis. The implications of these new findings could mean that the colonisation of the skin is not very diverse in this group of heifers.

\section{Conclusions}

None of the healthy heifers exposed to DD infected animals developed DD, evaluated both on a clinical or histopathological level. In only one of the 5 infected heifers the lesions remained unchanged during the study, the rest of the lesions healed.

In this study the clinical appearance of lesions (lesion stage) is not significantly associated with the prevalence of Treponema spp., the histopathological changes in epidermis and the level of inflammation in dermis. One of variables histopathological changes in epidermis or the level of inflammation in dermis (but not both) is significantly associated with the prevalence of Treponema spp. The effects of histopathological changes in epidermis and the level of inflammation in dermis on the prevalence of Treponema spp. have more or less the same positive directions: the likelihood of the prevalence of Treponema spp. increases with increasing level of histopathological changes in epidermis and severity of the inflammatory response.

It should be stressed that the number of observations is quite small for this study and that many of the results are just borderline significant.

All faecal samples collected rectally from the healthy heifers were positive for Treponema spp. on real-time PCR.

\section{Acknowledgements}

The study was funded by the Danish Research Council for Technology and Production. We thank the farmers for letting us borrow the cows.

\section{REFERENCES}

[1] N. Capion, S. M. Thamsborg and C. Enevoldsen, "Prevalence of Foot Lesions in Danish Holstein Cows," Veterinary Record, Vol. 163, No. 3, 2008, pp. 80-85. doi:10.1136/vr.163.3.80

[2] N. J. Evans, J. M. Brown, I. Demirkan, R. D. Murray, W. D. Vink, R. Blowey, C. A. Hart and S. D. Carter, "Three Unique Groups of Spirochetes Isolated from Digital Dermatitis Lesions in UK Cattle," Veterinary Microbiology, Vol. 130, No. 1-2, 2008, pp. 141-150. doi:10.1016/j.vetmic.2007.12.019

[3] C. M. Schroeder, K. W. Parlor, T. L. Marsh, N. K. Ames, A. K. Goeman and R. D. Walker, "Characterization of the Predominant Anaerobic Bacterium Recovered from Digital Dermatitis Lesions in Three Michigan Dairy Cows," Anaerobe, Vol. 9, No. 3, 2003, pp. 151-155. doi:10.1016/S1075-9964(03)00084-2

[4] D. J. Trott, M. R. Moeller, R. L. Zuerner, J. P. Goff, W. R. Waters, D. P. Alt, R. L. Walker and M. J. Wannemuehler, "Characterization of Treponema Phagedenis-Like Spirochetes Isolated from Papillomatous Digital Dermatitis Lesions in Dairy Cattle," Journal of Clinical Microbiology, Vol. 41, No. 6, 2003, pp. 2522-2529. doi:10.1128/JCM.41.6.2522-2529.2003

[5] M. Nordhoff, A. Moter, K. Schrank and L. H. Wieler, "High Prevalence of Treponemes in Bovine Digital Dermatitis-A Molecular Epidemiology," Veterinary Microbiology, Vol. 131, No. 3-4, 2008, pp. 293-300. doi:10.1016/j.vetmic.2008.04.019

[6] F J. N. Evans, J. M. Brown, I. Demirkan, P. Singh, B. Getty, D. Timofte, D. Vink, R. D. Murray, R. W. Blowey, 
R. Birtles, C. A. Hart and S. D. Carter, "Association of Unique, Isolated Treponemes with Bovine Digital Dermatitis Lesions," Journal of Clinical Microbiology, Vol. 47, No. 3, 2009, pp. 689-696. doi:10.1128/JCM.01914-08

[7] T. Yano, R. Yamagami, K. Misumi, C. Kubota, K. K. Moe, T. Hayashi, K. Yoshitani, O. Ohtake and N. Misawa, "Genetic Heterogeneity among Strains of Treponema Phagedenis-Like Spirochetes Isolated from Dairy Cattle with Papillomatous Digital Dermatitis in Japan," Journal of Clincal Microbiology, Vol. 47, No. 3, 2009, pp. 727733. doi:10.1128/JCM.01574-08

[8] K. Klitgaard, M. Boye, N. Capion and T. K. Jensen, "Evidence of Multiple Treponema Phylotypes Involved in Bovine Digital Dermatitis as Shown by 16S rRNA Gene Analysis and Fluorescence In Situ Hybridization," Journal of Clinical Microbiology, Vol. 46, No. 9, 2008, pp. 3012-3020. doi:10.1128/JCM.00670-08

[9] M. Rasmussen, N. Capion, K. Klitgaard, T. Rogdo, T. Fjeldass, M. Boye and T. K. Jensen, "Bovine Digital Dermatitis: Possible Pathogenic Consortium Consisting of Dichelobacter nodosus and Multiple Treponema Species," Veterinary Mircobiology, Vol. 160, No. 1-2, 2012, pp. 151-161. doi:10.1016/j.vetmic.2012.05.018

[10] B. K. Choi, H. Nattermann, S. Grund, W. Haider and U. B. Gobel, "Spirochetes from Digital Dermatitis Lesions in Cattle Are Closely Related to Treponemes Associated with Human Periodontitis," International Journal of Systematic Bacteriology, Vol. 47, No. 1, 1997, pp. 175-181. doi:10.1099/00207713-47-1-175

[11] R. Laven, "Control of Digital Dermatitis in Cattle," In Practice, Vol. 23, No. 6, 2001, pp. 336-341. doi:10.1136/inpract.23.6.336

[12] H. F. Bassett, M. Monaghan, P. Lenhan, M. L. Doherty and M. E. Carter, "Bovine Digital Dermatitis," Veterinary Record, Vol. 126, 1990, pp. 164-165.

[13] D. H. Read and R. L. Walker, "Papillomatous Digital Dermatitis (Footwarts) in California Dairy Cattle: Clinical and Gross Pathologic Findings," Journal of Veterinary Diagnostic Investigation, Vol. 10, No. 1, 1998, pp. 67-76. doi:10.1177/104063879801000112

[14] A. Gomez, N. Cook, N. D. Bernardoni, J. Rieman, A. F. Dusick, R. Hartshorn, M. T. Socha, D. H. Read and D. Döpfer, "An Experimental Infection Model to Induce Digital Dermatitis Infection in Cattle," Journal of Dairy Science, Vol. 95, No. 4, 2012, pp. 1821-1830. doi:10.3168/jds.2011-4754

[15] J. N. Evans, J. M. Brown, R. D. Murray, B. Getty, R. Birtles, C. A. Hart and S. D. Carter, "Characterization of Novel Bovine Gastrointestinal Tract Treponema Isolates and Comparison with Bovine Digital Dermatitis Treponemes," Applied and Environmental Microbiology, Vol. 77, No. 1, 2011, pp. 138-147. doi:10.1128/AEM.00993-10
[16] T. Shibahara, T. Ohya, R. Ishii, Y. Ogihara, T. Maeda, Y. Ishikawa and K. Kadota, "Concurrent Spirochaetal Infections of the Feet and Colon of Cattle in Japan," Australian Veterinary Journal, Vol. 80, No. 8, 2002, pp. 497-502. doi:10.1111/j.1751-0813.2002.tb12474.x

[17] T. B. Stanton and E. Canale-Parola, "Treponema Bryantii sp. nov., a Rumen Spirochete That Interacts with Cellulolytic Bacteria," Archives of Microbiology, Vol. 127, No. 2, 1980, pp. 145-156. doi:10.1007/BF00428018

[18] B. J. Paster and E. Canale-Parola, “Treponema Saccharophilum sp. nov., a Large Pectinolytic Spirochete from the Bovine Rumen," Applied Environmental Microbiology, Vol. 50, No. 2, 1985, pp. 212-219.

[19] D. J. Argaez-Rodriguez, D. W. Hird, J. H. deAnda, D. H. Read and A. Rodriguez-Lainz, "Papillomatous Digital Dermatitis on a Commercial Dairy Farm in Mexicali, Mexico: Incidence and Effect on Reproduction and Milk Production," Preventive Veterinary Medicine, Vol. 32, No. 3-4, 1997, pp. 275-286. doi:10.1016/S0167-5877(97)00031-7

[20] M. Holzhauer, C. Hardenberg, C. J. M. Bartels and K. Frankena, "Herd- and Cow-Level Prevalence of Digital Dermatitis in the Netherlands and Associated Risk Factors," Journal of Dairy Science, Vol. 89, No. 2, 2006, pp. 580-588. doi:10.3168/jds.S0022-0302(06)72121-X

[21] A. Rodriguez-Lainz, P. Melendez-Retamal, D. W. Hird, D. H. Read and R. L. Walker, "Farm- and Host-Level Risk Factors for Papillomatous Digital Dermatitis in Chilean Dairy Cattle," Preventive Veterinary Medicine, Vol. 42, No. 2, 1999, pp. 87-97. doi:10.1016/S0167-5877(99)00067-7

[22] A. Rodriguez-Lainz, D. W. Hird, R. L. Walker and D. H. Read, "Papillomatous Digital Dermatitis in 458 Dairies," Journal of the American Veterinary Medical Association, Vol. 209, No. 8, 1996, pp. 1464-1467.

[23] D. J. Sprecher, D. E. Hostetler and J. B. Kaneene, "A Lameness Scoring System that uses Posture and Gait to Predict Dairy Cattle Reproductive Performance," Theriogenology, Vol. 47, No. 6, 1997, pp. 1179-1187. doi:10.1016/S0093-691X(97)00098-8

[24] D. Döpfer, A. Koopmans, F. A. Meijer, I. Szakall, Y. H. W. Schukken, W. Klee, R. B. Bosma, J. L. Cornelisse, A. J. A. M. van Asten and A. A. H. M. ter Huurne, "Histological and Bacteriological Evaluation of Digital Dermatitis in Cattle, with Special Reference to Spirochaetes and Campylobacter Faecalis," Veterinary Record, Vol. 140, No. 24, 1997, pp. 620-623. doi:10.1136/vr.140.24.620

[25] N. Capion, M. Boye, C. T. Ekstrøm and T. K. Jensen, "Infection Dynamics of Digital Dermatiits in First-Lactation Holstein Cows in an Infected Herd," Journal of Dairy Science, Vol. 95, No. 11, 2012, pp. 1-8. doi:10.3168/jds.2012-5335 